\title{
CURRENT TREATMENT OF TRIGEMINAL NEURALGIA
}

\author{
Guenther Lanner und Martin C. Spendel \\ Clinic of Neurosurgery Klagenfurt, Klinikum Klagenfurt, Austria Klagenfurt, A - 9026 Klagenfurt, Austria
}

\section{Introduction}

The first step in the treatment of trigeminal neuralgia is conservative treatment with carbamazepin, gabapentin or other antiepileptic drugs. If medications have proven ineffective or presence of side effects or other drawbacks surgical treatment is necessary. According to literature and our own experiences in 1520 operated patients about half of all patients require an operation for pain relief.

\section{Microvascular Decompression}

A milestone in the treatment of trigeminal neuralgia was the understanding of vascular compression in the root-entry-zone of trigeminal nerve, described as early as 1929 by Dandy, rediscovered in 1959 by Gardner and popularized in 1976 by Jannetta.

Diagnostic criteria: MR -studies are necessary to disclose symptomatic trigeminal neuralgia like demyelination plaques of multiple sclerosis, as well as tumors and arteriovenous malformations in the cerebello-pontine angle.

MR-angiography with a special technique may show in almost all cases a compression and a deformity of trigeminal nerve in the root-entry-zone by a vascular structure.

Operation: At the operation by Jannetta the compressing vessel is separated from the trigeminal nerve, mobilized and replaced. Between the nerve and vessel a piece of muscle or Teflon is implanted.

Clinical Results: During a 15 years period $(1996$ - 2011) 428 patients underwent a microvascular decompression.

The mean follow-up was 9,8 years with arrange of $1-15$ years.
Immediately after microvascular decompression 99\% ( 423 ), up to 1 year $98 \%$ ( 419 )

and after 12 and 15 years 92\% (393) were free of pain. 35 patients had partial relief and required medication, 11 of them additionally underwent percutaneous radiofrequency rhizotomy.

Negative predictors for this operation are symptoms lasting more than 10 years $(p<0,001)$ and previous ablative treatment $(p<0,006)$. In our study there was a total rate of complications of $2 \%$. Most complications were transitory: hypacusis, paresis of facial nerve, CSF -fistula.

Conclusion: Mikrovascular decompression is the only causal operative procedure and method of choice in cardiopulmonary healthy patients without(!) cut-off age.

The advantages of the microvascular decompression compared to ablative operations are beside the excellent postoperative results the intact function of the trigeminal nerve, no hyp-,dysand paresthesia as well as no anesthesia dolorosa.

It is a safe and effective method with low morbidity, mortality and excellent long-term results.

\section{Radiofrequency Rhizotomy}

In the literature and in our experiences the indication of radiofrequency rhizotomy

Is determined by high age with bad condition and simultaneous internal deseases.

In the last 20 years we have performed thermocoagulation of the Gasserian ganglion in 584 patients.

Technique: Our approach to the foramen ovale and method of 
electrode placement is similar to that described by Sweet in 1969. In local anesthesia the foramen ovale is punctured by use of X-ray control and the thermosound is placed into the Gasserian ganglion. To evaluate the position of the thermosound we performed motor and sensory stimulation. In short general anesthesia the rhizotomy is then conducted.

Clinical results: The immediate postoperative results in $584 \mathrm{pa}-$ tients with a mean follow up of 14,6 years $(1-20$ a $)$ are excellent, we had an immediate pain relief in $95 \%$ of the patients. In the postoperative controls after 5, 10 and 20 years these excellent results decreased and after 10 years $80 \%$ and after 20 years $75 \%$ of the patients were free of pain.

The recurrence rate was after 5 years $12 \%$ and after 10 years $20 \%$.

Conclusion: The advantages of radiofrequency rhizotomy compared to other procedures are the short period of operation, branch selection of trigeminal nerve by multiple control of the needle position, a low morbidity , no limit due to age, the high rate of success, the possibility of repetition and a short stay in the hospital.

\section{Stereotactic Radiosurgery}

Method: In trigeminal neuralgia a single highly concentrated dose of radiation is directed to a small, precise target at the trigeminal root, producing delayed injury to nervous tissue

Results: In the literature and in 32 own patients there was a primary success rate of $70-90 \%$, after 5 years only $55 \%$ of the patients were free of pain.

Radiosurgery of trigeminal neuralgia has as a disadvantage a delayed effect on pain reduction not until weeks or months and as a side effect homolateral paresthesias, dose related in 3-10\%.

In summary, it can be stated that all these surgical procedures are complementary methods. Operative treatment requires exactly selection of patients and precise technique of operation. 